\title{
A Fast Approach to Perform Susceptibility Measurement in A Stepped-mode Reverberation Chamber
}

\author{
Lingyuan Meng \\ Mechanical Engineering college \\ Shijiazhuang, China \\ fclwdtf@163.com \\ Guanghui Wei \\ Mechanical Engineering college \\ Shijiazhuang, China
}

\author{
Xiaodong Pan \\ Mechanical Engineering college \\ Shijiazhuang, China \\ Haojiang Wan \\ Mechanical Engineering college \\ Shijiazhuang, China
}

\begin{abstract}
On the base of analysis of probability of failure under uncertainty of susceptibility measurement in the reverberation chamber, we derive the relationship between the sample number of stirrer positions and the number of the mean value of electric field of different injected power levels during the measurement, the duration of measurement will reduce to a certain extent by select appropriate parameters. Combined on the two different approach( substitution approach and removing approach) to represent the real field strength suffered on the devices putting in the reverberation chamber based on the statistic characteristics of reverberation chamber, We propose a fast approach to perform susceptibility measurement in a stepped-mode reverberation chamber. Such an approach can be apply for a much wider range of susceptibility measurement compared with other testing environment and other approach in reverberation chamber.
\end{abstract}

Keywords-reverberation chamber; susceptibility measurement; duration; field strength; statistic characteristics

\section{INTRODUCTION}

Susceptibility measurement, as an important issue on EMC, more and more attention is attracted. it has made much progress on susceptibility measurement performed in OTAS ( Open testing area space), GTEM cell and FAR ( fully anechoic chamber). However, the testing electronic field offered is either deterministic or random, the impinging maximum electronic field of these testing environment can't reach the susceptibility level, the reverberation chamber (RC) is an electrically large , high-Q rectangular cavity, who obtains statistically isotropic, randomly polarized and uniform electromagnetic field within an acceptable uncertainty and confidence limit [1], who can easily offer environment of great magnitude electronic field, and statistical mode theories have been used to analyze reverberation chamber. Compared with other testing area, much advantages performing susceptibility measurement are obvious [2]:

A large testing electronic field with moderate input power;
b)To finish the test, the EUT ( equipment under testing) don't need to rotate, and the antenna don't need to change the direction of polarization;

c) To decline the construction cost;

d)To perform the susceptibility measurement of system level;

There are much international standards of measurement in reverberation chamber, like MIL-STD 461E [3] and the International Electrotechnical Commission 61000-4-21 [4]. There are two work modes of reverberation chamber, continuous stirred-mode operation and stepped-mode operation, the standard suggested we to choose the stepped-mode operation which the EUTs have enough time to respond to the testing environment.In stirred operation, the tuner is continuously rotated while the measurements are performed, with no stops at specific angles. The stirring exposes the EUT to the maximum number of field configurations possible within a given chamber at the operating frequency when the tuner is rotated through a full turn. However, a concern with mechanical stirring is that the EUT may not be exposed to the required field level for a sufficient length of time to provide full susceptibility measurement, it's much difficult to make sure the specific electric field value impinging on the EUTs [5]. Compared with stirrer-mode operation, the duration of the measurement is an important issue. Measurements must be delayed until these mechanically induced transients die down. We can derive the duration $\mathrm{T}$ given by [6]:

$$
T=N\left(t+n n_{E} t_{E}\right)+2 \pi / \omega
$$

When the $N$ is the sample measured,which is also the number of independent stirrer position, $n$ is the number of frequency used during the measurement, $t$ is the time needed for the stabilization of the mechanical stirrer when the step by step motor stops, when change the injected power level, the value of E-field in the RC changes, until the EUTs break down and $n_{E}$ is the number of E-field in RC with different injected power levels, $t_{E}$ is the duration of the testing 
for one injected power level under one frequency, and $\omega$ is the angular speed of the stirrer.

To decrease the duration of the test $T$, we propose a possible approach to reduce the $n_{E}$ or $N$. In this article, we find the relationship between the number of E-field in RC with different injected power levels $n_{E}$ and the stirrer positions $N$, to decrease $T$ by choosing the appropriate $n_{E}$ and $N$.

\section{ANALYSIS}

A. The statistic analysis of field distribution in $R C$

As a random free field,the distribution everywhere in reverberation chamber is independent, the real and imaginary part of the resulting electric field in an ideal reverberation chamber follow a normal distribution with zero mean and $\sigma^{2}$ variance,take $\mathrm{x}$ rectangular component for an example, it's written in terms of his real and imaginary part by [7]:

$$
\left|E_{x}\right|=\sqrt{\left[N\left(0, \sigma^{2}\right)\right]^{2}+\left[j N\left(0, \sigma^{2}\right)\right]^{2}}
$$

The magnitude of any of the electric field components is distributed with two degrees of freedom and consequently has a Rayleigh distribution, the PDF(Probability Distribution Function) is given:

$$
f_{X}\left(E_{x}\right)=\frac{E_{x}}{\sigma^{2}} \mathrm{e}^{-\frac{E_{x}^{2}}{2 \sigma^{2}}}, E_{x} \geq 0
$$

Therefore, the mean value of E-field in reverberation chamber $E_{m}$ is:

$$
E_{m}=\sigma \sqrt{\pi / 2}
$$

Do the integral by $\left(0, E_{x}\right]$, we can derive the CDF(Cumulative Distribution Function):

$$
F_{X}\left(E_{x}\right)=\int_{0}^{E_{x}} \frac{x}{\sigma^{2}} \exp \left(-\frac{x^{2}}{2 \sigma^{2}}\right) d x=1-\mathrm{e}^{-\frac{E_{x}^{2}}{2 \sigma^{2}}}
$$

Considering the susceptibility level of EUT is $E_{0}$, when the value of E-field $E_{\text {in reverberation chamber }}$ exceeds $E_{0}$, The EUT observe a failure at one stirrer position.. This probability $p$ is given:

$$
p\left(E \geq E_{0}\right)=1-F_{X}\left(E_{0}\right)=\mathrm{e}^{-\frac{E_{0}{ }^{2}}{2 \sigma^{2}}}
$$

Thus,

$$
p=e^{-\frac{\pi^{2} E_{0}{ }^{2}}{4 E_{m}^{2}}}
$$

We can derive the $E_{0}$ as the function of $p$ and $E_{m}$, it's written by:

$$
E_{0}=E_{m} \sqrt{\frac{4 \ln (1 / p)}{\pi}}
$$

B. The possibility of failure $p$

The susceptibility level of an EUT generally follow a binomial distribution, when the value of E-field suffered by EUT exceeds the susceptibility level, the EUT breaks down. If $p$ is the possibility of failure in the measurement, $x$ is a random variable, the possibility of $m$ failures among $n$ times testing is written by:

$$
P(x=m)=C_{n}^{m} p^{m}(1-p)^{n-m}
$$

Derivation $P$ for $p$

$\frac{d P}{d p}=(n-m p) \frac{n !}{m !(n-m) !} p^{m-1}(1-p)^{n-m-1}$

When $m=n p$, the value of $P$ gets the maximum, therefore when perform $n$ times susceptibility measurement, $m$ failures is recorded, the estimation of the possibility of failure $p$ is written by :

$$
\not=m / n
$$

\section{Confidence interval}

Considering a binomial population probability $p$, whose distribution is approximated by the normal distribution 0 mean and variance equal to 1 . According to the Wilson Interval, which has good properties especially for a small number of trials, under the confidence level of $Z_{1-\alpha / 2}$, the confidence interval of the possibility of failure $p$ is written by [8]:

$$
\begin{aligned}
& p_{\min }=\frac{1}{1+\frac{Z_{1-\alpha / 2}^{2}}{n}}\left[\not p+\frac{Z_{1-\alpha / 2}^{2}}{2 n}-Z_{1-\alpha / 2} \sqrt{\frac{\not p(1-\not p)}{n}+\frac{Z_{1-\alpha / 2}^{2}}{4 n^{2}}}\right] \\
& p_{\max }=\frac{1}{1+\frac{Z_{1-\alpha / 2}{ }^{2}}{n}}\left[\not p+\frac{Z_{1-\alpha / 2}^{2}}{2 n}+Z_{1-\alpha / 2} \sqrt{\frac{\not(1-\not p)}{n}+\frac{Z_{1-\alpha / 2}^{2}}{4 n^{2}}}\right]
\end{aligned}
$$

By using (1), we can derive the estimation of the susceptibility level of EUT $\mathbb{E}_{0}$, which is written by [9] :

$$
\mathbb{E}_{0}=E_{m} \sqrt{\frac{4 \ln (1 / \not p)}{\pi}}
$$

By using the Wilson interval, under the confidence level of $Z_{1-\alpha / 2}$, the confidence interval of the susceptibility level $E_{0}$ of EUT is written by:

$$
\left[E_{m} \sqrt{\frac{4 \ln \left(1 / p_{\max }\right)}{\pi}}, E_{m} \sqrt{\frac{4 \ln \left(1 / p_{\min }\right)}{\pi}}\right]
$$




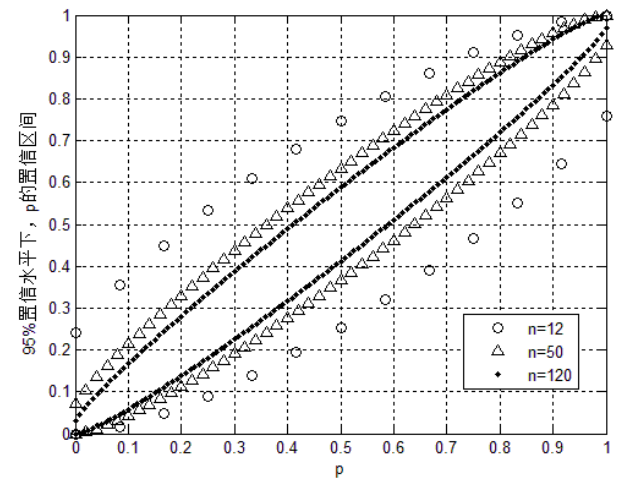

Figure 1. The confidence interval of $p$ under the confidence level of $95 \%$, when $n$ is 12,50 , and 120 .

Let $E_{m_{i}}$ denotes the mean value of the E-field in reverberation chamber on $i$ th testing power level, and $E_{m_{i+1}}$, which is the mean value of the electric field in reverberation chamber on the successive testing power level, can be written by:

$$
E_{m_{i+1}} \approx E_{m_{i}} \sqrt{\frac{\ln p_{\min }}{\ln p_{\max }}}
$$

Thus, if we choose $E_{m_{1}}$ as the mean value of electric field in reverberation chamber on the first testing injected power level, the $E_{m_{i}}$ can be derived from (16) by:

$$
E_{m_{i}}=E_{m_{1}}\left(\sqrt{\frac{\ln p_{\min }}{\ln p_{\max }}}\right)^{i-1}
$$

We define $\rho$ for the correlation coefficient between $E_{m_{1}}$ and $E_{m_{i}}$ :

$$
E_{m_{i}}=E_{m_{1}}(\rho)^{i-1}
$$

This is how to calculate $\rho$ from different $n$ and $p$ under the confidence interval of $Z_{1-\alpha / 2}$ :

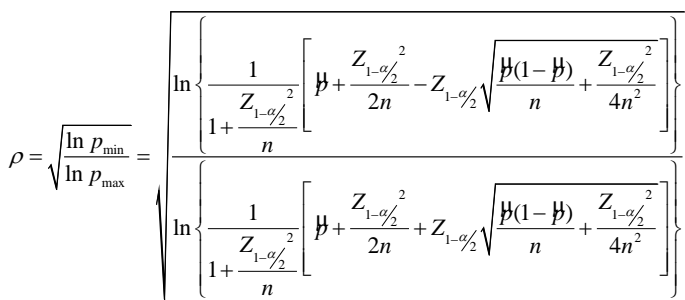

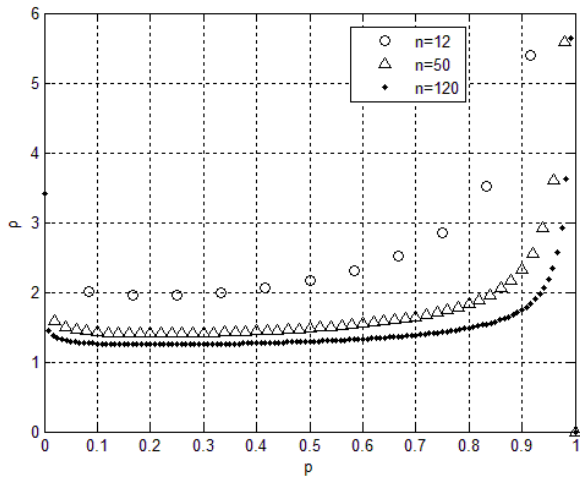

Figure 2. The value of $\rho$ under the confidence level of $95 \%$, when $n$ is 12,50 and 120 .

Therefore, when we have the interval of the estimation of susceptibility level $\left[E_{0-}, E_{0+}\right]$ indicated by the mean value of electric field suffered in the EUT, take the minimum $E_{0-}$ as the first mean value $E_{m_{1}}$, while take the maximum $E_{0+}$ as the last mean value, thus as the number of electric field in RC with different injected power levels, $n_{E}$ is derive from (18), which is written by:

$$
n_{E}=\text { Ceiling }\left[\log _{\rho} \frac{E_{0+}}{E_{0-}}+1\right]
$$

Ceiling denotes the integer round up to the latter. Therefore, it's certain expression between $n_{E}$ and $N$, when we have the estimation of $\left[E_{0-}, E_{0+}\right]$ and the susceptibility properties of EUT denoted by the probability of failure during the test.

\section{MEASUREMENT}

When we put EUTs into the reverberation chamber, the electric field change, the mean value of field strength in a loading reverberation chamber with devices is different with the empty reverberation chamber, the field around the devices is the superimposed field of the incident field and the scattering field by the devices[10]. Thus, the measurement of electric field strength attracts more and more interest, there we put forward two different methods to perform the measurement.

A. $\quad$ substitution method

Based on the characterization of statistically isotropic, randomly polarized and uniform electromagnetic field of reverberation chamber, select multiple measurement

positions, the stirrer(s) rotates and record the field strength on certain one measurement position of multiple stirrer positions, or take the stirrer on one position and record the field strength of multiple measurement positions, use the field strength recorded to represent the electric field suffered on EUTs by specific statistical analysis approach.

$B$. removing method 
When the EUT is electronic small enough which the size is negligible compared with the wavelength, holding the stirrer on one position, and, measure the field strength before and after putting the EUT into the reverberation chamber on the position which the EUTs (will ) put on and the referential position which is appropriate enough around the EUT, derive the loading coefficient of reverberation chamber by comparing the normalized field strength on the referential position, then confirm the real field strength suffered on the device by using the loading coefficient derived.

\section{CONCLUSION}

This article aims at to obtain the relationship between the sample measured,which is also the number of independent stirrer position, and the number of electric field in reverberation chamber with different injected power levels by studying the properties of the probability of the failure during the susceptibility measurement, by reducing the sample number or injected power levels to decrease the measurement time and improve the measurement efficiency. We show that equation, it's easily to get the appropriate number under the uncertain level of susceptibility measurement.

Another aspect to measure the exact field strength suffered on the EUTs, we provide two approach according the reverberation chamber characteristics of statistically isotropic, randomly polarized and uniform. Removing method is aimed at the device of small electronic size, and it's more accurate than the substitution method, while when we put an EUT of large electronic size compared with wavelength, the substitution method is much more convenient than the removing method.

\section{REFERENCES}

[1] David A. Hill, "Electronic Mode Stirring for Reverberation Chambers [J]," IEEE Transactions on Electromagnetic Compatibility, vol. 36, no. 4, Nov. 1994, pp.294-299, doi:10.1109/15.328858.

[2] Li zhang, Fugui Shen, and Yuming Hu, "A Discussion on the effct of loading absorbing material on the Reverberation Chamber [C]," International Conference on Remote Sensing, Environment and Transportation Engineering (RSETE) in Nanjing,Jun. 2011, pp 959-962, doi:10.1109/RSETE.2011.5964438.

[3] Requirements for the Control of Electromagnetics Interference Charac-teristics of Subsystems and Equipments [S], MIL-STD-461F, 2007.

[4] Electromagnetic Compatibility (EMC). Part 4-21: Testing and Measurements Techniques-Reverberation Chamber Methods [S], IEC 61000-4-21,2011.

[5] Vignesh Rajamani, Charles F. Bunting, "Stirred-Mode Operation of Reverberation Chambers for EMC Testing [J]," IEEE Transactions on Instrumentation and Measurement", vol. 62, no. 10, Oct. 2012, pp. 2759-2764. Doi:10.1109/TIM.2012.2196398.

[6] Emmanuel Amador, Christophe Lemoine, and Philippe Besnier, "Optimization of Immunity Testing in a Mode Tuned Reverberation Chamber with Monte Carlo Simulations [D]," Proceedings ESA Workshop on Aerospace EMC in Venice, May 2012, pp. 1-6.

[7] David A. Hill, "Plane Wave Integral Representation for Fields in Reverberation Chambers [J]," IEEE Transactions on Electromagnetic Compatibility, vol. 40, no. 3, Aug. 1998, pp. 209-217, doi:10.1109/15.709418.

[8] E. B. Wilson, "Probable inference, the law of succession, and statistical inference[J]," J. Amer. Statist. Ass., vol. 22, no. 158, 1927, pp. 209-212.

[9] Emmanuel Amador, Hans Georg Krauthauser, and Philippe Besnier, "A Binomial Model for Radiated Immunity Measurements [J]," IEEE Transactions on Electromagnetic Compatibility, vol. 55, no. 4, 2015, pp. 683-691, doi: 10.1109/TEMC.2012.2231942.

[10] Nick Janssen, Kate A. Remley, and Christopher L. Holloway, "Correlation coefficient and loading effects for MIMO antennas in a reverberation chamber [D]", International Symposium on Electromagnetic Compatibility (EMC EUROPE), Sep. 2013, pp. 514-519. 